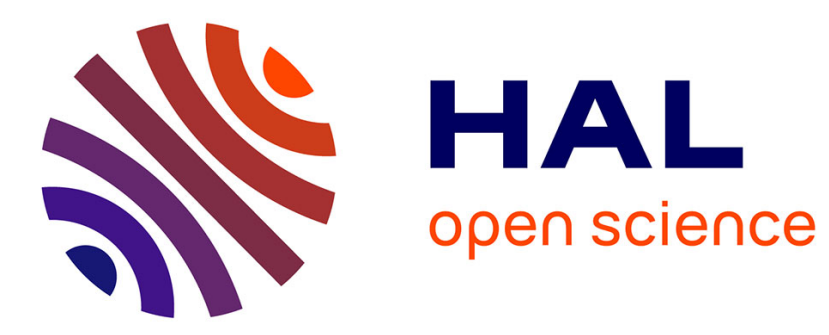

\title{
Chemically limited versus diffusion limited aggregation
}

M. Kolb, R. Jullien

\section{To cite this version:}

M. Kolb, R. Jullien. Chemically limited versus diffusion limited aggregation. Journal de Physique Lettres, 1984, 45 (20), pp.977-981. 10.1051/jphyslet:019840045020097700 . jpa-00232439

\section{HAL Id: jpa-00232439 https://hal.science/jpa-00232439}

Submitted on 1 Jan 1984

HAL is a multi-disciplinary open access archive for the deposit and dissemination of scientific research documents, whether they are published or not. The documents may come from teaching and research institutions in France or abroad, or from public or private research centers.
L'archive ouverte pluridisciplinaire HAL, est destinée au dépôt et à la diffusion de documents scientifiques de niveau recherche, publiés ou non, émanant des établissements d'enseignement et de recherche français ou étrangers, des laboratoires publics ou privés. 
Classification

Physics Abstracts

$05.50-64.60$

\title{
Chemically limited versus diffusion limited aggregation
}

\author{
M. Kolb and R. Jullien \\ Laboratoire de Physique des Solides $\left({ }^{*}\right)$, Bât. 510, Université Paris-Sud, \\ Centre d'Orsay, 91405 Orsay Cedex, France
}

(Reçu le 18 juin 1984, accepté le 27 août 1984)

\begin{abstract}
Résumé. - Dans des processus réalistes de croissance, les facteurs cinétiques et chimiques déterminent la structure des agrégats. Nous proposons un modèle qui tient compte de ces deux facteurs. Nous déterminons l'exposant fractal de l'agrégation chimique $D=1,55 \pm 0,05(D=2,00 \pm 0,08)$ en deux (trois) dimensions. On étudie le comportement de crossover entre les régimes d'agrégations chimique et diffusive.
\end{abstract}

\begin{abstract}
In realistic growth processes, both kinetic and chemical factors determine the structure of the aggregates. Here, a model is proposed which interpolates between a purely diffusive and a purely chemical situation. There is crossover from a new, chemically limited growth model, with fractal exponents $D=1.55 \pm 0.05(2.00 \pm 0.08)$ in two (three) dimensions, to diffusive cluster aggregation.
\end{abstract}

Geometrical properties of clusters observed in aggregation have been investigated intensively in recent years, both experimentally and theoretically. Simple descriptions in terms of scaling have lead to a characterization of these clusters in terms of fractal objects, whereby the growth mechanism is instrumental in determining the fractal exponent.

A situation, which is of particular interest, is the growth in aerosols (smoke formation) and in colloids, where diffusion plays an important rôle. Direct measurements [1-4] have produced evidence that the aggregates are quite ramified and do have scaling properties. Theoretically, a description in terms of an irreversible, purely kinetic process has been proposed $[5,6]$.

In realistic situations, a number of different effects compete to determine the characteristics of the clusters grown. Besides the influence of the kinetic motion, the chemical properties such as the number of active molecules will have to be considered. Furthermore, the aggregates tend to restructure during the growth, which certainly modifies their appearance. An example, where all these effects blend together is the macroscopic experiment of wax balls floating on a water surface [7].

When comparing the fractal exponents of the kinetic clustering of clusters model $(D \cong 1.78$ in three dimensions) with experiments, one finds that the model may explain some experiments $(D \cong 1.75$ in Ref. [2]) but differs clearly in other cases $(D \cong 2.12,2.52$ in Refs. [3, 4]). This motivates us to generalize the diffusive growth model to allow for chemical effects. To do this, we introduce a sticking probability $p$ as an extra parameter into the diffusion limited model $[5,6]$.

(*) Laboratoire associé au C.N.R.S. 
As $p \rightarrow 0$, a new chemically limited model, with a different fractal exponent characterizes the clusters. For finite $p$, there is crossover from the chemically limited to the diffusion limited model (Fig. 1).

The model, which we wish to investigate numerically, is the following : a number of clusters diffuse randomly in a periodically bounded box. Two clusters which touch each other during their motion, can form a permanent bond at the point of contact with probability $p$. If they do form a bond, they form a new, larger cluster, which also diffuses through the box. If the bond is not formed, the two clusters continue to diffuse independently, but without overlapping each other. As the growth is irreversible, the remaining clusters gradually become bigger and bigger. The mobility of the clusters is assumed to depend on their mass as

$$
v=m^{\alpha}
$$

where the exponent $\alpha$ is characteristic of the kinetic mechanism. This model is a generalization of clustering of clusters $[5,6]$ with $p$ as an additional parameter. The actual study has been performed under similar conditions as in reference [6] : the clusters are placed on a hypercubic lattice, jump one lattice spacing at a time and "touch " when they are nearest neighbours. The initial configuration is a collection of randomly distributed single particles, and one is interested in the regime of low particle concentration.

The parameter $p$ in our model determines to what extent diffusive resp. chemical bonding dominates the aggregation process. The two limits $p=1$ and $p=0$ correspond to diffusion limited and chemically limited aggregation. For $p=1$, as studied in references $[5,6]$, the growth is controlled effectively by the screening of the outer tips of the clusters, which prevents them from penetrating each other beyond a characteristic length $\xi$ and leads to very ramified structures; see table I. In the limit when $p \rightarrow 0$, two clusters touch each other many times before they form a bond. Hence, the outer portions no longer prevent them from penetrating each other. As in cluster aggregation the clusters have typically the same size, there is nevertheless a steric constraint on how far they can penetrate. This suggests that the resulting structures are more compact than in the diffusive case but still have a non trivial fractal dimension $(D<d)$. Thus, we define purely chemically limited aggregation as the process, where two coalescing clusters have the same probability to form a chemical bond between any molecule of the first cluster and any molecule of the second cluster, but respecting the steric constraint, that the clusters cannot overlap. We have calculated the fractal dimension of this model by directly applying this procedure to a collection of clusters (initially each of one particle). The rôle of $\alpha$

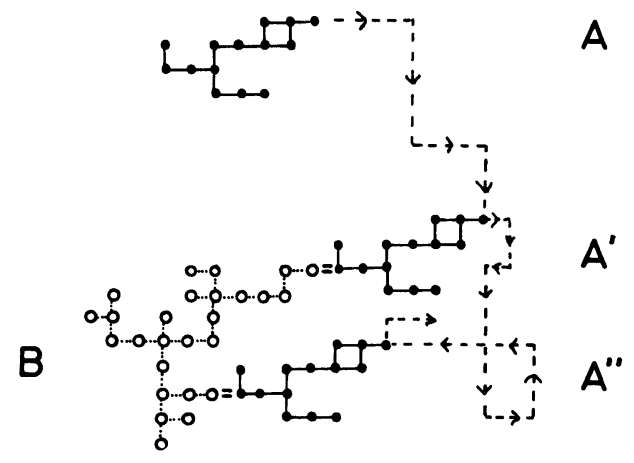

Fig. 1. - Sketch of diffusion process. Two clusters diffuse (dotted line, one cluster held fixed) until they touch $\left(\mathrm{A} \rightarrow \mathrm{A}^{\prime}\right)$. A bond is formed with probability $p$. If not, the diffusion continues (no overlap of the clusters) until the clusters touch again $\left(A^{\prime} \rightarrow A^{\prime \prime}\right)$, etc... 
(Eq. (1)), is now played by $\omega$ in

$$
K_{m_{1} m_{2}}=\left(m_{1} m_{2}\right)^{\omega}
$$

where $K_{m_{1} m_{2}}$ is the probability that a cluster with $m_{1}$ particles links up with a cluster, with $m_{2}$ particles. Thus we pick a pair of clusters with $m_{1}$ resp. $m_{2}$ particles with probability $K_{m_{1} m_{2}}$ out of our collection and then bond them with the same probability in one of the sterically allowed ways. The alternative, hierarchical version of this model is treated in detail in reference [8], where additionally the number of chemically active points is considered.

Guided by the results for $p=1[6,9]$, one expects that the fractal exponent varies with the dimension, but - to within numerical errors - is the same for the simulations in the box $(\alpha \approx 0, p \simeq 0)$, for the collection of clusters $(\omega \approx 0)$ and for the hierarchical version (both clusters have the same mass). The following slight differences between the models also do not appear to have an influence on the fractal properties : 1) holes and bottlenecks are chemically inactive in the box, they are not excluded in the case of the collection of clusters ; 2) when two clusters simultaneously touch at two (or more) points, the probability to bind can be either taken to be $p$ or $2 p$.

In analogy with particle aggregation, this $p$-dependent model interpolates between a model with kinetic dimension $d_{\mathrm{w}}=2$ and $d_{\mathrm{w}}=0$ [10]. The limits $p=1$ and $p=0$ for particle aggregation correspond to the diffusion limited aggregation model and the Eden model.

The intermediate situation where $0<p<1$ can be viewed qualitatively as follows. Before two clusters form a chemical bond, they have a number $(\sim 1 / p)$ of contacts. We separate the process in two parts : first there is a diffusion limited motion until the clusters touch for the first time. The characteristic interpenetration length $\xi$ of the two clusters grows with their radius $R$ (the simplest scaling theory predicts that these two lengths are proportional). After the first contact, the aggregation can be formulated differently: how far do two ramified clusters that initially touch each other penetrate before touching $1 / p-1$ more times ? This is determined by the local diffusion around the initial point of contact for ramified objects. The characteristic distance $\xi_{0}\left(\sim p^{-1 / 2}\right)$ then does not depend on $R$. For large clusters $\xi-\xi_{0} \sim \xi$ and there is effectively still diffusion limited screening. Thus one expects for small $p$ and large $R$ a crossover from chemical to diffusive aggregation.

We have numerically investigated the model with and without a box and varying $\alpha$ resp. $\omega$ $(₹ 0)$. In figure 2 clusters with $p=1$ and $p=0$ are compared. The latter is clearly more compact than the former, but still ramified. In table I the exponents for chemically limited aggregation are presented and compared with diffusive aggregation. Above the upper critical dimension, where the clusters become transparent $\left(d_{\mathrm{c}}=2 D_{\mathrm{c}}+d_{\mathrm{w}}, D_{\mathrm{c}}=3.4\right.$ [1]) there is no distinction between the two models. The results for chemical aggregation are found to be independent of $\omega$ to within the numerical errors for $\omega \lesssim 0$ similar to diffusive aggregation $[6,9]$. The exponents

Table I. - Fractal exponent D for chemical aggregation. The values are obtained for purely chemical aggregation and are insensitive to $\omega(\lesssim 0)$. For comparison, the corresponding values for diffusive aggregation [6, 9] are added. The error bars (statistical error) are $\sim \pm 0.06$.

\begin{tabular}{|c|c|c|}
\hline$d$ & $\begin{array}{c}D \\
\text { chemical }\end{array}$ & $\begin{array}{c}D \\
\text { diffusive }\end{array}$ \\
\hline 2 & 1.55 & 1.42 \\
3 & 2.00 & 1.78 \\
4 & 2.32 & 2.04 \\
\hline
\end{tabular}




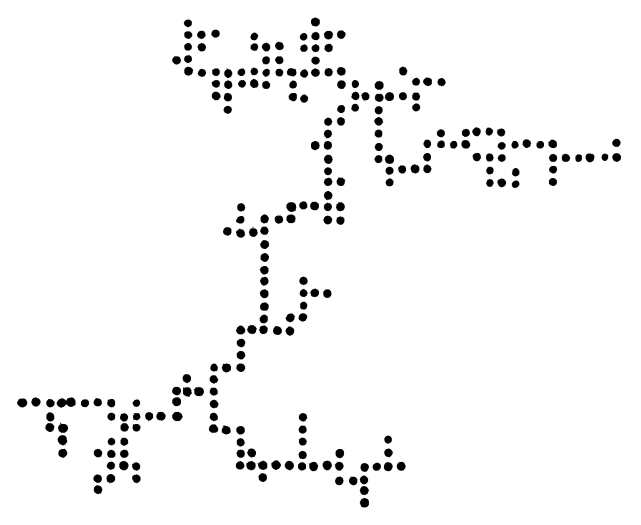

\section{diffusive}

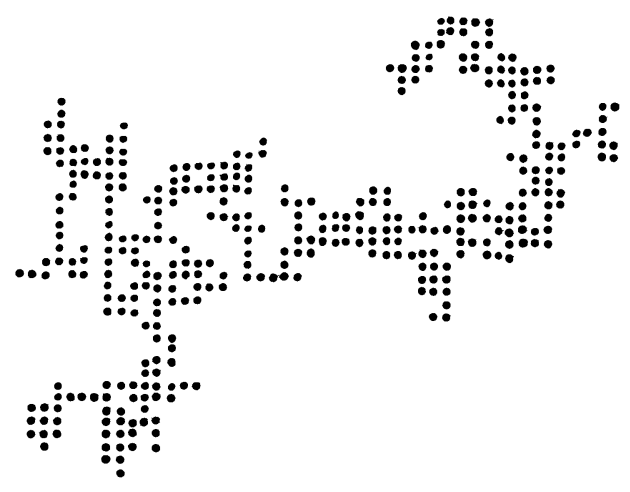

\section{chemical}

Fig. 2. - Diffusion limited cluster $(p=1)$ and chemically limited cluster $(p=0)$. The latter is more compact than the former, but still ramified.

were calculated from the data for the radius of gyration $m \sim R^{D} . D_{\text {eff }}=\ln (R(2 m) / R(m)) / \ln (2)$ for successive (average) values of $m$ are extrapolated to $m \rightarrow \infty$ to estimate $D$. In $d=2$, for example, $D_{\text {eff }}=1.32(02), 1.48(04), 1.50(05), 1.51(05), 1.53(05)$ for $m=4,8,16,32,64$ and similarly in higher dimensions.

To illustrate the crossover, we have calculated $\frac{R(p=1)}{R(p)} v s . m$ logarithmically for a number of values of $p$ in two dimensions. Figure 3 is consistent with a crossover from the chemical to the diffusive case.

In experimental situations, the exact nature of the kinetic mechanism and the chemical bonding relevant for the aggregation are not known. This may explain, why the experiments [1-4] yield different exponents for the aggregates. Nevertheless it is suggestive to compare the three dimen- 


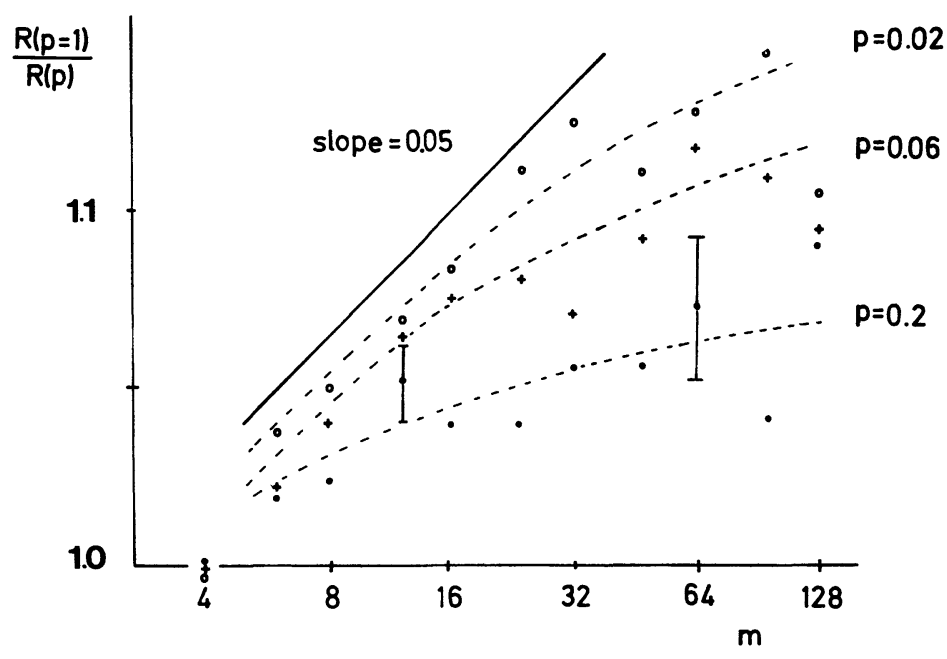

Fig. 3. - The ratio of the radii $R(p=1) / R(p)$ versus mass $m$ is plotted (log-log) for simulations in a box of length $L=128$ with 1024 particles and with $\alpha=0$. The slope drawn represents an estimate of the difference between the exponents $v(p=1)-v(p), v=1 / D$ (see table I). Despite the strong fluctuations one observes a trend that for small $p(\Theta,+, O$ correspond to $p=0.2,0.06,0.02$ respectively) the clusters grow chemically (slope $\sim 0.05$ ) before crossing over to the diffusive regime (slope $=0$ ). Crossover implies that $m(R)$ can be written as $R^{D_{0}} f\left(R / \xi_{0}\right), \xi_{0}=p^{-1 / 2}$. The numerical accuracy is not sufficient to determine $f(x)$.

sional experiments by Weitz $(D=1.75)$ and Schaefer et al. $(D=2.12)$ with the models discussed here. Of course, other factors may play a rôle, notably more complicated kinetics and restructuring.

\section{Acknowledgments.}

We acknowledge interesting discussions with $\mathbf{R}$. Ball and $\mathbf{R}$. Botet.

\section{References}

[1] Forrest, S. R. and Witten, T. A., J. Phys. A 12 (1979) L109.

[2] Weitz, D. A. and Oliveria, M., Phys. Rev. Lett. 52 (1984) 1433.

[3] Schaefer, D. W., Martin, J. E., Wiltzius, P. and Cannell, D. S., Phys. Rev. Lett. to appear (1984).

[4] Sinha, S. K., FreltofT, T. and KJEMS, J., in Kinetics of aggregation and gelation, Eds., D. P. Landau and F. Family (North Holland) 1984, to appear.

[5] Meakin, P., Phys. Rev. Lett. 51 (1983) 1119.

[6] Kolb, M., Botet, R. and Jullien, R., Phys. Rev. Lett. 51 (1983) 1123.

[7] Allain, C. and Jounier, B., J. Physique Lett. 44 (1983) L-421.

[8] Jullien, R. and KolB, M., unpublished (1984).

[9] Jullien, R., Kolb, M. and Botet, R., J. Physique Lett. 45 (1984) L-211.

[10] Meakin, P., Phys. Rev. A 27 (1983) 604.

[11] Ball, R. and Witten, T. A., Proceedings of the third conference on fractals, Gaitherburg, Md to be published in J. Stat. Phys. 